\title{
Stigma-stop a serious game against the stigma in mental disorders
}

\author{
Jose A. Piedra-Fernandez \\ Dpt. Computer Science \\ University of Almeria \\ Almeria, Spain \\ Email: jpiedra@ual.es
}

\author{
Adolfo J. Cangas \\ Dpt. Psychology \\ University of Almeria \\ Almeria, Spain \\ Email:ajcangas@ual.es
}

\author{
Juan Jesus Ojeda-Castelo \\ Alborada Engineers
}

Almeria. Spain

Email: castelock@gmail.com

\author{
Diego Cangas \\ Alborada Engineers \\ Almeria. Spain \\ Email: diegocangas6@gmail.com
}

\author{
Antonio J. Fernandez-Garcia \\ Dpt. Computer Science \\ University of Almeria \\ Almeria, Spain \\ Email:ajfernandez@ual.es
}

\begin{abstract}
In this work, a serious game is presented to sensitize young people to stigma in mental health. This a video game, developed by a multidisciplinary team (computer scientists, psychologists, psychiatrists and educators), features characters that have different mental disorders. It provides different information about what mental illness is while the player interacts with the characters in different ways, indicating which the most appropriate reactions are. There are also various short-games aimed at eradicating stigma. The game will be available for free to teenagers and college students. Furthermore, it can be used both independently and as part of other existing programs in schools against stigma of mental disorders.
\end{abstract}

\section{Introduction}

Today, one of the factors that most influences slow recovery in people with mental disorders is the stigma they experience. In this regard, there is a great deal of misinformation, misconceptions, prejudices, etc. about what the problems of mental health are. As a result, the general population remains quite distant with this group; they do not understand them and even reject them [1], [2].

These stigmatizing ideas are even common in college students, even in degrees such as Medicine or Psychology, Education, etc., which makes it necessary to include specific information on this issue in the training of university students [3], [4].

On the other hand, adolescence is a time of multiple personal, physical and social changes. It is precisely when the most serious mental disorders arise (such as schizophrenia, anorexia, substance abuse, etc.). Also, with regard to young people, there is significant level of ignorance and concern for problems related to depression, anxiety, etc. Hence, it is essential to carry out information campaigns that provide them with information and tools so they can face challenges and deal with these issues, which may be quite serious to them personally.
The most common way of working on these issues in education is through talks and workshops, often given by people who have suffered mental disorders. These individuals explain their experiences to young people, talk about the problems they faced, how the solved them, etc. In some cases, these experiences are accompanied by a video to introduce and explain the issue and discuss it. In short, they are intended to raise awareness about mental disorders, better understand their origins and what resources or methods exist to treat them.

These workshops help young people who are suffering, or are not sure whether they have some type of mental disorder, to answer numerous personal questions. For example, they offer information that might allow an individual to overcome their particular problem. Similarly, peers teach them to better understand the people who suffer these difficulties, to empathize with them, and show how they can help.

What has not been so common in these information campaigns is the use of video games, despite being a fun method, most commonly used by young people. This may be due to the high cost of developing this type of software, the need to form multidisciplinary teams to design them (computer scientists, psychologists, educators, etc.), or the absence of validated programs at this moment. Whichever the case, its use has been anything but widespread [5].

The aim of this project was precisely to develop a video game called Stigma-Stop, to sensitize young people about the characteristics of mental disorders and the ways of dealing with them. This is a project funded by the Government of Andalusia, developed by the spin-off of the University of Almeria Alborada Engineers and has the support of the Andalusian Public Federation for Social Integration of People with Mental Disorders, FAISEM; FEAFES-Andalusia Mental Health and, internationally, with Pacific Clinics (Los Angeles, USA).

This is a video game in which the characters are people that suffer from different mental disorders (agoraphobia, depression, bipolar disorder and schizophrenia). The pro- 
tagonist has to overcome a challenge, which is to form a team to design a video game to participate in a contest. The protagonist has to convince the various characters to contribute towards this goal. To do so, the program provides information on what these mental disorders may be due to and gives several options for interacting with the characters. If not done correctly, the player is offered feedback on why a certain action is not adequate and is given the opportunity to select other options.

Also, the player is going asking to interact with the characters, if you ever felt like them, at what times and what he has done. The aim is to sensitize young people to the difficulties of these individuals, to show that their experiences are not so different from those that we all deal with at times in our lives, and, ultimately, to demonstrate that young people themselves can also help others with these difficulties.

The video game also includes four short-games (see Figure 1) related to mental health. In one of them, players have to make pairs of famous people (see Figure 1a) who have suffered various mental disorders (musicians, scientists, writers, etc.), such as Charles Darwin, Leonardo Da Vinci, Edgar Allan Poe, Salvador Dali, etc. In all these cases, information is given to players about the mental disorders that these famous characters suffered from in order to highlight the idea that, despite their difficulties, people with mental illness can also make great contributions to humanity.

In the second short-game players must differentiate and destroy concepts which stigmatize mental disorders (see Figure 1b), such as ideas that these individuals are lazy, incurable, unpredictable, etc. Players earn points by gathering more appropriate ideas, such as acceptance, independence, achievement, etc. Another short-game is based on a trivia format (see Figure 1c) regarding knowledge they have about mental disorders and the stigmatizing thoughts towards them. Finally, the fourth short-game (see Figure 1d) features an obstacle course in which a painter has to sort different objects to reach his muse.

Thus, the objectives to be achieved with Stigma-Stop are the following:

- Give information to students about mental disorders.

- Encourage empathy towards people with mental illness.

- Banish misconceptions about mental illness.

- Highlight biographical aspects in mental illness (which help participants to understand people with mental illness and put themselves in their place).

- Highlight the contextual issues that precipitate or facilitate the development of mental disorders (stressful situations, social situations, isolation, etc.).

- Emphasize the idea that anyone can suffer a mental disorder.

- Emphasize the idea that everyone can have (more or less) similar experiences that a person with a mental disorder.

- Encourage the normalization of these experiences, that is, show ways to react less alarmed or more naturally to a person with a mental disorder.

- Change the idea that the behavior of people with mental illness is always unpredictable.

- To emphasize that, despite the difficulties, they can achieve goals.

Stigma-Stop can be played both on the computer or in an App, which is currently being developed as most teenagers have a mobile device [7]. At the moment, there is a version in Spanish, but there are also plans to develop one in English.

The use of Stigma-Stop is free and can be used both independently and in conjunction with other programs that are taught in schools against stigma of mental disorders.

\section{Description of the Game}

The video game was developed by two platforms with the game engine Unity3D [6]: web and mobile app. The server manages a database for storing relevant data of the game, such as the responses to the forms, score achieved or information from the user, such as age or sex. Communication between the server and the two types of clients (see Figure 2) is carried out by web services in JSON requests, and server access your MySQL database through PHP to provide the client with the corresponding response, for instance, that the sent data has been inserted correctly.

At the beginning of the game the player can choose between a male or female character, depending on user preference, with the aim of involving the user and making the experience more personal and realistic.

The main goal is to visit every one of your friends, each of whom has a different mental illness. In the video game, the player can approach any of the characters (Figure 3), not knowing in advance which illness each has. In this way, the game is not based on a linear plot that forces you to do certain actions. This game consists of a series of branches (one per character) that lead to the same ending: meet each of the mental disorders and manage to submit the video game to the contest.

The gamer can choose any character rendered on the board, just clicking on the picture of the corresponding character. Once a character has been completed, this character cannot be selected. In fact, when the player returns, they are shown the option to choose the next friend. Characters that have already been previously selected will appear deactivated and marked with a green tick.

The interface (see Figure 4) is mainly divided into three panels: a panel at the bottom showing the goal to be achieved (Figure 4a), a panel at the top indicating the action the user has to perform (Figure 4b) and the short-map (Figure 5)in the top right. This short-map indicates the location of the character in the scene with a blue circle and a red arrow points to the objective, which has to address the main character.

There are some parts of the game in which the user can interact (i.e., choosing a friend, going for a walk around the city or filling out a form) and move the avatar in different 


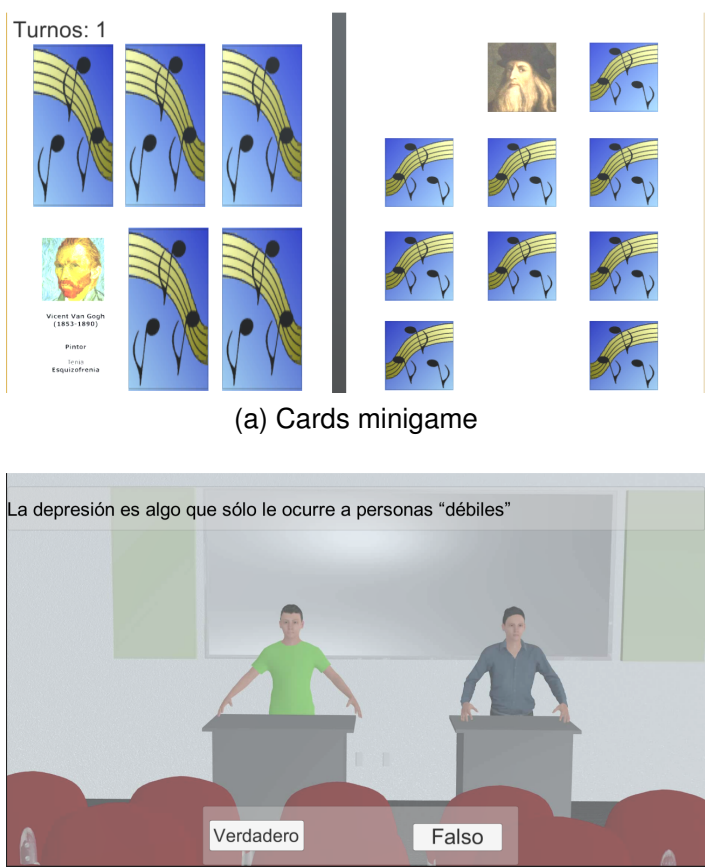

(c) Trivial minigame

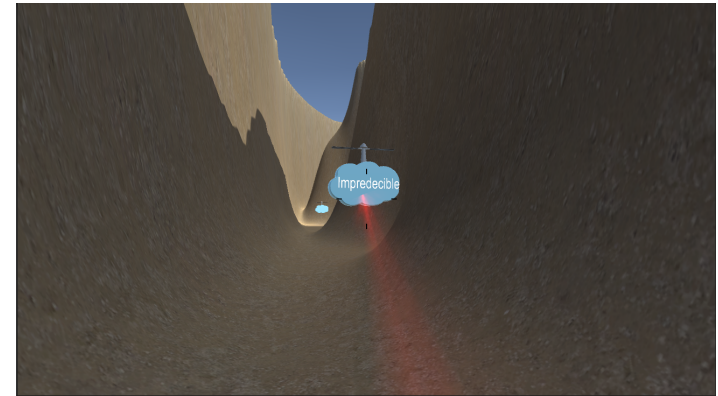

(b) Stigmatizing concepts minigame

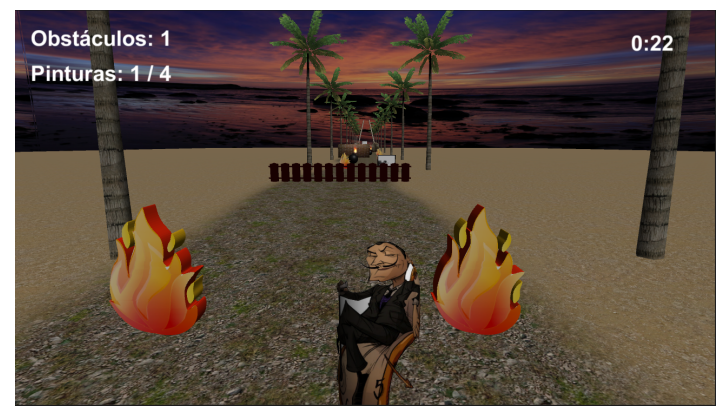

(d) Running minigame

Figure 1. The minigames.

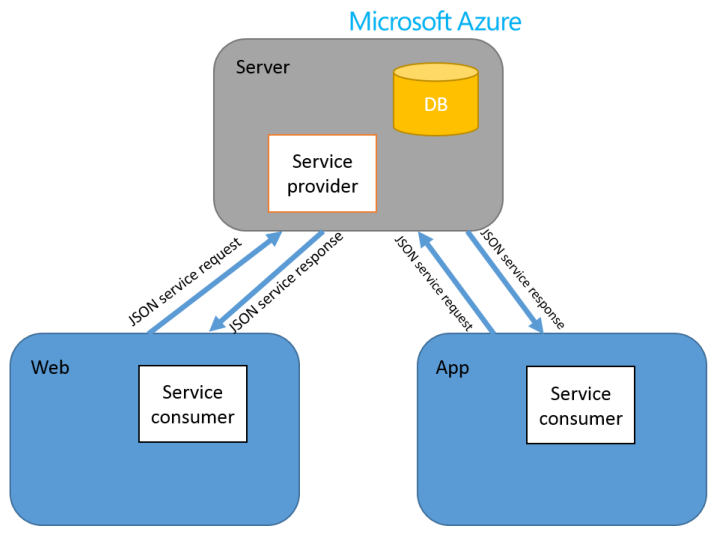

Figure 2. Architecture scheme.

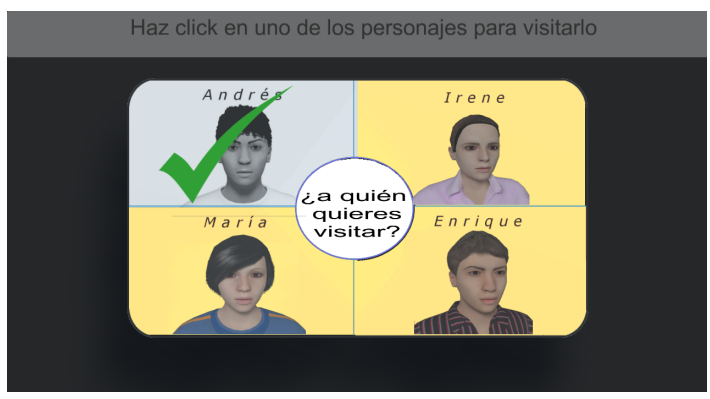

Figure 3. Choice of the character. scenarios of the game. However, when the user gets to one of the friends, a dialog begins with the character by means of animation. At this stage of the game some symptoms of the disease have been shown and when the main line of the dialog has finished, the user can choose from three options (see Figure 6). Only one of the options is correct. When any of the options are selected, an animation of dialog showing the result of the chosen action will be played. If the player chooses the wrong option, the options screen will be displayed but the previously-selected option will be crossed out and an explanation of why that reaction was wrong in the given situation will be provided.

In addition, the user has to fill in a form (Figure 7). On the left side of this form a series of questions will be shown that the user will answer, and on the right side the user may access more information about the current status of the characters and what circumstances led them to develop this type of anomaly.

\section{Conclusion and Future Work}

The video game was developed by the opinion of adolescents about mental illness and how they would react to a person who suffers from this disorder. The video game guides them on the most appropriate behavior in these situations. The players know in more detail the four types of mental illness listed in the introduction and learn to empathize with people who suffer from these problems.

A web application was developed so as to be accessible to the greatest number of people, regardless of place of 


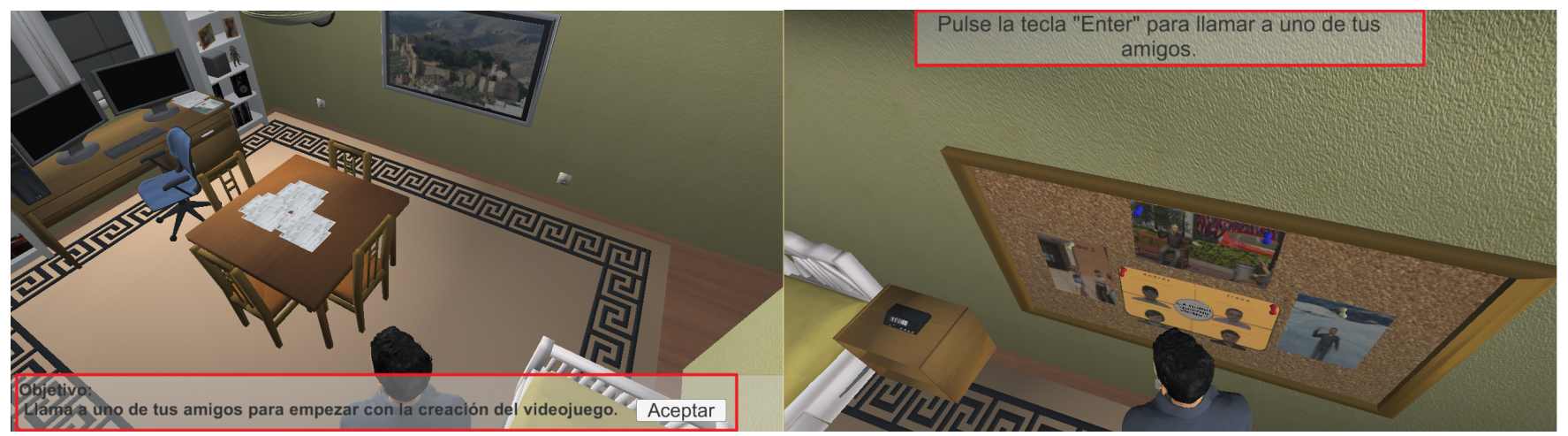

(a) Panel of goals

(b) Panel of actions

Figure 4. Panels of the interface.

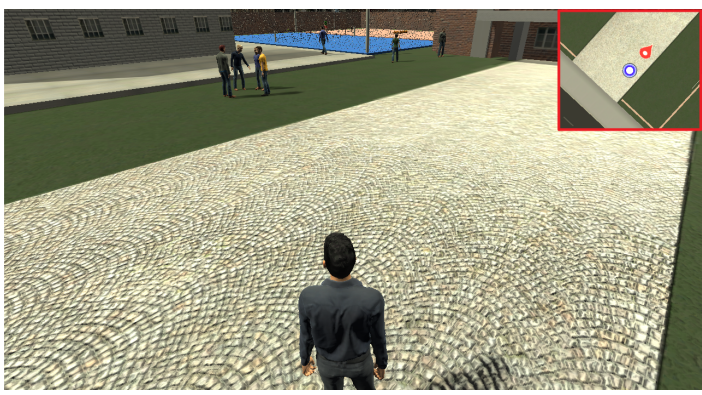

Figure 5. The short-map of the game.

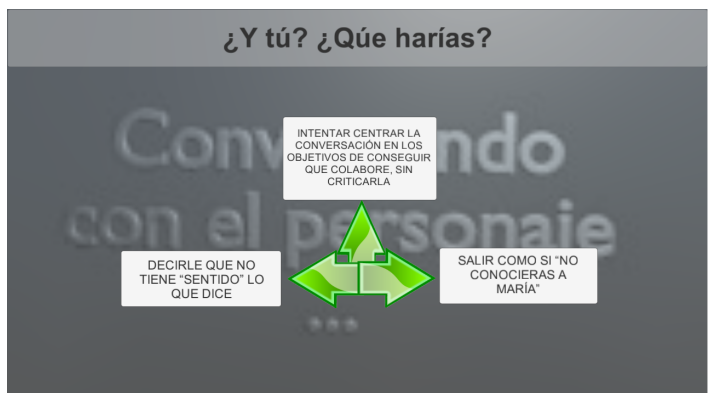

Figure 6. Choice of actions.

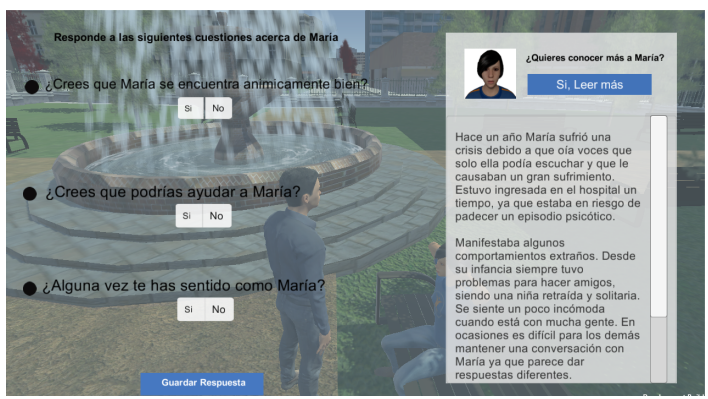

Figure 7. Questionnaire about a character.

residence. The purpose is that it can be used internationally and, therefore, a version in English is being created, which largely justifies the decision to create an online video game. A mobile application is under development, since the majority of young people now have a mobile device. It is easy for them to install the application on their personal device and they will be able to play anywhere.

Future work will look into enhancing this experience by using virtual reality headsets and exploring the benefits and drawbacks.

\section{Acknowledgments}

This work was funded by the Regional Government of Andalusia (Excellence Research Projects (SEJ-7204) and the EU ERDF and the Spanish Ministry of Economy and Competitiveness (MINECO) under Projects TIN2013-41576-R and the Andalusian Regional Government (Spain) under Project P10-TIC-6114. This work also received funding from the CEiA3 and CEIMAR consortiums.

\section{References}

[1] PJ. Michaels, M. Lpez , N. Rsch, PW. Corrigan, Constructs and concepts comprising the stigma of mental illness, Psyc So Edu, vol.4, pp. 183-194, 2012.

[2] A. Masuda, M. Hill, J. Morgan, L. L. Cohen, A psychological flexibility-based intervention for modulating the impact of stigma and prejudice: a descriptive review of empirical evidence, Psyc So Edu, vol. 4, pp. 211-223, 2012.

[3] C. Galletly, C. Burton, Improving medical student attitudes towards people with schizophrenia. Aust Nz J Psychiat, vol. 45, pp. 473-476, 2011.

[4] PW. Corrigan, SB Morris, P.J Michaels, J.D. Rafacz, N. Rsch. Challenging the public stigma of mental Illness: A meta-analysis of outcome studies. Psychiatric Services, 63, 963-973, 2012.

[5] J.M. Mateu, N. Navarro. Key and evidences of the use of ICT in severe mental disorder, Psycho Soc Edu, Vol. 7, pp. 85-95, 2015.

[6] Unity Technologies, Unity - Game Engine. Published online https: //unity3d.com/, March 2016.

[7] Strasburger, V. C., Hogan, M. J., Mulligan, D. A., Ameenuddin, N., Christakis, D. A., Cross, C., ... and Moreno, M. A. Children, adolescents, and the media. Pediatrics, vol.132, no. 5, pp. 958-961,2 013. 